\title{
Actualización en estenorraquis lumbar: diagnóstico, tratamiento y controversias
}

\author{
MARCELO MOLINA, PABLO WAGNER, MAURICIO CAMPOS
}

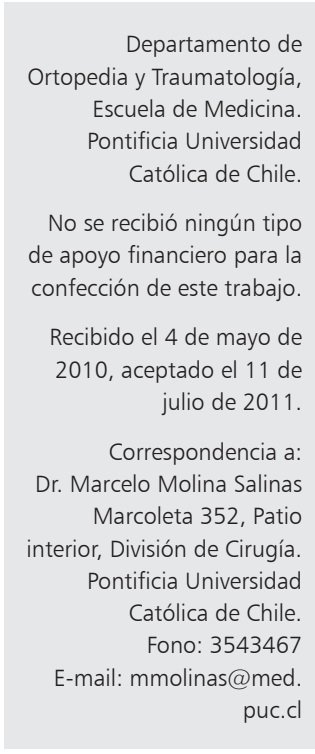

\section{Spinal lumbar stenosis. An update}

Spinal lumbar stenosis is a disease that occurs mainly between the $5^{\text {th }}$ and $7^{\text {th }}$ decade of life and can be congenital or acquired. The latter has many etiologies, but a degenerative cause is the most common. Stenosis is a narrowing of the spinal canal diameter caused by many factors such as bulging discs, hypertrophy of flavum ligament, facet capsule thickening and osteophyte formation. The classical symptom of the disease is sciatic pain, that improves with lumbar flexion and worsens with ambulation. Neurological examination is often normal and the most useful imaging test is magnetic resonance imaging (MRI). Conservative management consists in non-steroidal anti-inflammatory drugs, muscle relaxants, physical therapy and epidural - radicular infiltrations. Spinal infiltrations have a proven efficacy for pain management. A good result of this therapy predicts a favorable outcome after surgery. Surgical treatment consists in decompression with or without lumbar fusion. The addition of an arthrodesis is recommended for degenerative spondylolisthesis, correction of deformities, recurrent spinal stenosis with instability, sagittal or coronal imbalance and adjacent segment disease.

(Rev Med Chile 2011; 139: 1488-1495).

Key words: Arthrodesis; Spinal stenosis; Spondylolisthesis.
$\mathrm{L}$

a raquiestenosis lumbar se define como una disminución del área del canal raquídeo que puede comprimir las raíces nerviosas. La incidencia en las imágenes de este cuadro va de $1,7 \%$ a $13,1 \%^{1}$, sin embargo, la incidencia clínica es menor y genera sus síntomas con mayor frecuencia entre la $5^{\circ}$ y la $7^{\circ}$ década de la vida. Su causa principal son los cambios degenerativos de la columna vertebral. Se produce con igual frecuencia entre hombres y mujeres, excepto cuando esta patología se asocia a espondilolistesis, la cual es de mayor incidencia en mujeres. No hay asociación con actividad laboral o física.

\section{Anatomía}

La columna vertebral está constituida por componentes óseos con sus ligamentos, articulaciones, discos y músculos. El raquis consta de 33 vértebras: 7 cervicales, 12 dorsales, 5 lumbares, 5 sacras (fusionadas) y el cóccix, que resulta de la unión de las 4 vértebras coccígeas. Las vértebras cervicales, dorsales y lumbares se articulan entre sí mediante los discos intervertebrales y las articulaciones facetarias.

Los elementos ligamentarios proporcionan una estructura estable y móvil. Entre los ligamentos de mayor significación figuran:

- Longitudinal anterior.

- Longitudinal posterior, que forma parte de la pared anterior del canal medular.

- Ligamento amarillo, que se insertan entre las láminas, e integran la pared posterior del canal raquídeo.

- Interespinosos y supraespinosos.

La unidad vertebral está constituida por 2 vertebras más los ligamentos, articulaciones facetarias y el disco intervertebral entre ambas vértebras. 
Respecto a los límites del canal raquídeo; estos constan de: por anterior los discos y cuerpos vertebrales, por posterior las facetas articulares y láminas. El diámetro anteroposterior del canal raquídeo tiene una media de $12 \mathrm{~mm}$ y el área necesaria para acomodar los elementos neurales es de $77 \pm 13 \mathrm{~mm}^{2-4}$. Sin embargo, más que un valor absoluto importa la relación entre el tamaño del canal y su contenido.

La estenosis del canal raquídeo puede ocurrir a nivel central, receso lateral (porción más lateral del canal), foraminal o extraforaminal.

Las estructuras anatómicas que producen estenorraquis son el disco intervertebral cuando este protruye hacia el canal (bulging), hipertrofia del ligamento amarillo, hipertrofia capsular facetaria, osteofitos facetarios y del cuerpo vertebral y dependiente de estructuras óseas cuando hay displasias que predisponen a un área menor del canal (Figuras 1,2)

\section{Fisiopatología}

Se describe una cascada de procesos llamada de Kirkaldy-Willis en honor a sus autores, que detalla y separa la patogenia en 3 etapas: Disfunción, inestabilidad y estabilización posterior.

Inicialmente ocurre una deshidratación del núcleo pulposo que conlleva a una pérdida de altura del disco. Estos cambios se producen por lo general en la tercera década de la vida. Esto produciría algún grado de inestabilidad segmentaria alterándose la transmisión de la carga. Más tardíamente se observan cambios a nivel de las articulaciones facetarias con engrosamiento de la cápsula y ligamentos que aparecen entre los 40 y 50 años lo que es secundario a la inestabilidad del segmento. Posteriormente se forman osteofitos tanto a nivel del cuerpo vertebral como de las facetas que tienden a estabilizar el segmento. Todo lo descrito previamente resulta en un estrechamiento de los diámetros del canal raquídeo y de las foráminas.

La estenorraquis puede asociarse además a espondilolistesis (desplazamiento en plano sagital de un cuerpo vertebral respecto al adyacente), escoliosis (desviación del eje de la columna en el plano coronal) o a inestabilidad. La clasificación más utilizada es la de Arnoldi (Tabla 1) que separa los tipos según su etiología en congénitas $\mathrm{y}$ adquiridas, siendo las estenorraquis adquiridas degenerativas la causa más frecuente.

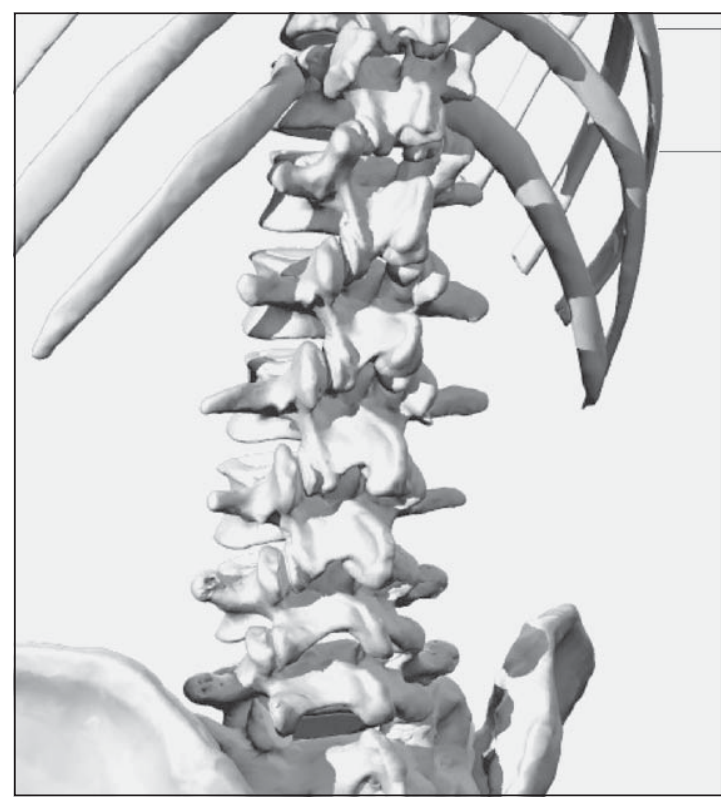

Figura 1. Columna lumbar: visión panorámica posterolateral.

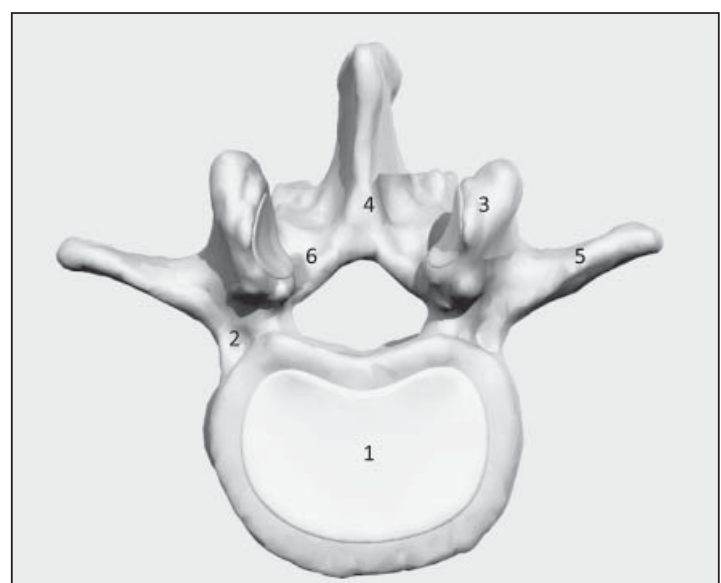

Figura 2. Vértebra lumbar: visión axial. 1: Cuerpo Vertebral; 2: Pedículos; 3: Articulaciones facetarios; 4: Arco posterior (incluye desde los pedículos hasta las apófisis espinosas); 5 : Apófisis transversa; 6: Láminas.

En relación a la patogenia del dolor, la compresión de las raíces puede provocar déficit sensitivo, motor y alteración de reflejos. Sin embargo, para producir dolor debe estar asociada a inflamación de la raíz. Aún no se sabe exactamente el mecanismo de dolor en la compresión neurológica, sin embargo, se postula que sería por un mecanismo 
Tabla 1. Clasificación de Arnoldi de estenorraquis

I. Congénita
a. Idiopática
b. Acondroplásica
c. Osteopetrosis
II. Adquirida
a. Degenerativa
i. Central
ii. Receso lateral y foraminal
iii. Espondilolistesis degenerativa
b latrogénica
i. Post-laminectomía
ii. Post-artrodesis
iii. Post-discectomía
c. Misceláneos
i. Acromegalia
ii. Enfermedad de Paget
iii. Fluorosis
iv. Espondilitis anquilosante
d. Traumática
Combinada I + II

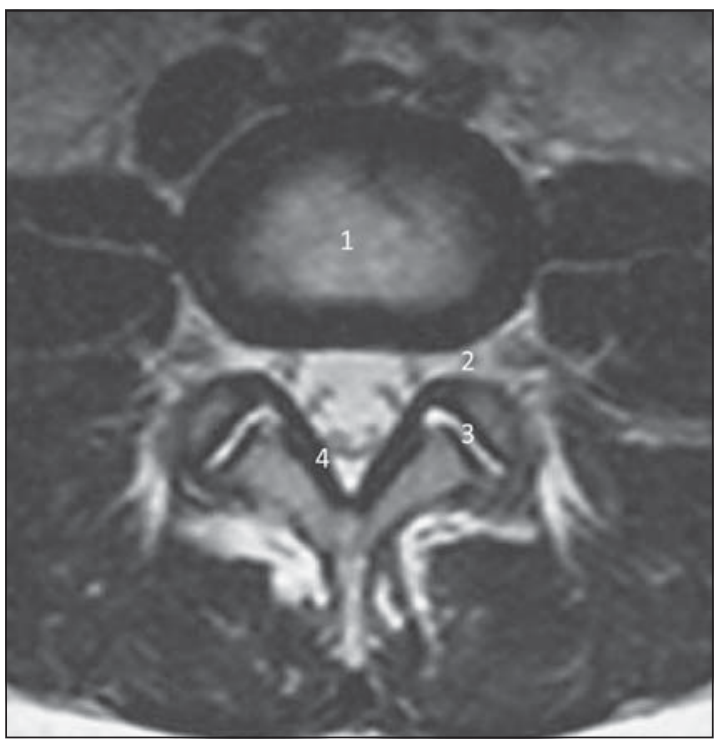

Figura 3. Vértebra lumbar: visión axial bajo Resonancia Magnética. 1: Disco intervertebral; 2: Forámenes Vertebrales; 3: Articulación facetaria; 4: Ligamento amarillo. en cadena, en el cual hay un aumento de la presión venosa, posterior edema y congestión de la estructura afectada, terminando en un cuadro "tipo síndrome compartimental".

\section{Cuadro clínico}

La estenorraquis lumbar puede ser asintomática. En estudios realizados en individuos asintomáticos a los cuales se les realiza una RM, se ha encontrado una prevalencia de hasta $20 \%$ de raquiestenosis degenerativa en el estrato de edad de los 60 a 80 años, siendo excepcional en pacientes más jóvenes ${ }^{5-8}$. Cuando es sintomática, el síntoma principal es el dolor lumbar y radicular'. El dolor radicular puede presentarse como una lumbocruralgia, que es cuando el dolor lumbar se irradia a la cara anterior del muslo o bien, como una lumbociática que es la irradiación del dolor bajo la rodilla, con frecuencia hasta el tobillo y pie. El curso clínico de la enfermedad es lento, insidioso, de larga evolución, de localización inicialmente lumbar, irradiándose luego a glúteos y finalmente a extremidades como una cruralgia o como una ciatalgia.

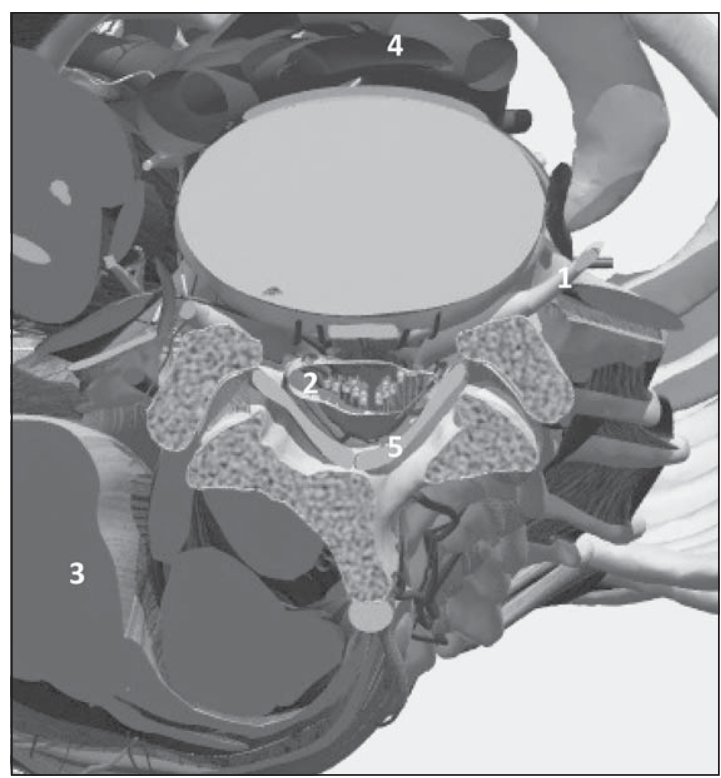

Figura 4. Vertebral lumbar: visión axial con anatomía periférica. 1: Raiz vertebral saliendo de formanen vertebral; 2: Saco dural con raíces en su interior (cola de caballo); 3 : Musculatura extensora de la columna; 4: Grandes vasos ilíacos; 5: ligamento amarillo. 
El cuadro clínico que produce al deambular se le denomina claudicación neurogénica. Este es un cuadro que se caracteriza por la aparición o aumento progresivo de dolor, parestesias o paresia en extremidades inferiores al caminar. Estos síntomas disminuyen o ceden con el reposo y con la flexión de columna. Debe diferenciarse de la claudicación vascular, la cual se caracteriza por síntomas parecidos, con dolor en extremidades inferiores que aumenta con la marcha, pero a diferencia de la claudicación neurogénica, no presenta alteraciones sensitivas ni motoras y no mejora con la flexión de columna. Clínicamente no siempre es clara esta diferencia, es más, en ocasiones ambas claudicaciones pueden coexistir en algunos pacientes, requiriendo un estudio vascular o de imágenes de columna para definir la patología presente o predominante.

Se debe destacar que habitualmente no compromete función esfinteriana rectal ni vesical. El compromiso de los elementos neurales es de tipo radicular. Los niveles más comprometidos son L3 - L4, L4 - L5 y L5 - S1.

Respecto a la frecuencia de los síntomas 9 , el dolor lumbar asociado al dolor ciático se encuentra en 95\% de los casos, claudicación de la marcha en 91\% y alteraciones neurológicas de las extremidades en al menos 70\%. El dolor radicular puede ser bilateral hasta en $42 \%$ de los casos.

\section{Examen físico}

El examen físico se debe realizar de forma exhaustiva. Se debe iniciar con el paciente de pie. Inicialmente se realiza la inspección en busca de las curvaturas normales y anormales de la columna (escoliosis, lordosis o cifosis) y evaluar el balance del tronco. Posterior a eso se procede al examen de la movilidad de la columna, examen neurológico segmentario radicular tanto motor, sensitivo como de evaluación de reflejos. Los signos de irritación radicular son habitualmente negativos. Estos son el TEPE (test de extensión con pierna estirada), Lasségue y la maniobra de Gowers-Bragard, todos los cuales se realizan en decúbito supino. El TEPE consiste en la elevación de la extremidad afectada con la rodilla en extensión. Es positivo si se reproduce el dolor radicular. El signo de Gowers-Bragard se realiza a continuación del TEPE, y consiste en disminuir la elevación de la extremidad extendida hasta que el dolor radicular ceda, realizándose posteriormente una dorsiflexión del pie. Es posi- tivo si genera dolor ciático con la dorsiflexión del pie. El Lasségue consiste en la flexión de la cadera a $90^{\circ}$ y posteriormente realizar una extensión de la rodilla. Es positivo si causa dolor radicular. Los recién nombrados son signos para la evaluación radicular lumbar baja (L4-S1). Para la irritación crural o lumbar alta (L1-L3) se debe buscar el signo de $\mathrm{O}^{\prime}$ Connell, que se explora en decúbito prono realizando una extensión de la extremidad afectada. Es positivo si se reproduce el dolor crural con la maniobra.

Parte del examen físico debe apuntar también a descartar los principales diagnósticos diferenciales de esta patología que son la claudicación vascular y el dolor proveniente de la cadera. Respecto a la claudicación vascular este cuadro consta principalmente de dolor en zona posterior de las piernas (pantorrillas), disminución de pulsos y temperatura distales, entre otros elementos. Respecto al dolor proveniente de la cadera este se debe investigar a través de los movimientos articulares en todo su rango de movimiento.

Por lo general el examen físico es normal. Una estrategia para encontrar ciertos elementos del cuadro descrito por el paciente es que realicen una caminata, para que así aparezcan molestias y posteriormente re-examinarlo.

\section{Estudio imagenológico}

Las imágenes por si solas no realizan diagnóstico, siendo este un complemento entre la clínica y las imágenes. En este sentido las radiografias, la tomografía computada (TAC) o la resonancia magnética (RM) siempre deben ser solicitadas para evaluar una sospecha clínica.

Respecto al estudio del paciente siempre debe iniciarse mediante radiografías convencionales de columna lumbar. Se debe realizar una radiografía tanto estática (antero posterior y lateral) como dinámica en flexión y extensión, que evidenciarán la estabilidad de la columna. Se define como inestabilidad un desplazamiento mayor a $4 \mathrm{~mm}$ o una angulación mayor a $10^{\circ}$ de una vértebra respecto a la otra. Se pueden apreciar también otras alteraciones como pedículos cortos (estenorraquis congénita), espondilolistesis, fracturas o tumores. Si se sospecha escoliosis, hipercifosis, hiperlordosis o alteración del balance sagital o coronal se debe tomar una radiografía de columna total.

La resonancia magnética es el estándar de oro para la confirmación del diagnóstico y evaluación 
preoperatoria. Se visualiza adecuadamente la hipertrofia facetaria, sinovitis facetaria, hipertrofia del ligamento amarillo y capsular, alteraciones discales (como protrusión, HNP o bulging) todo lo cual puede producir estenosis central, de recesos laterales o foráminas. Entre otras cosas permite evidenciar la presencia de otros procesos como tumores o infecciones. Antiguamente la instrumentación producía importante artefactos en las imágenes, pero la instrumentación más reciente con tornillos pediculares y barras de titanio produce menos artefactos en las imágenes lo que permite evaluar pacientes post operado.

Respecto a la TAC (sin contraste), ampliamente solicitada en nuestro país ante la sospecha de esta patología, tiene menor definición y nitidez para evaluar partes blandas, raíces nerviosas y ligamentos. Con este examen se logra identificar con claridad los osteofitos e hipertrofia ósea que contribuyen a la estrechez del canal o forámina.

La TAC con mielografía es una opción válida y útil en pacientes con instrumentación axial en los cuales no se puede realizar un RM. Permite definir los elementos óseos, neurológicos y delimitar el canal raquídeo para evaluar una estenosis. Se aprecian bien los recesos laterales, hipertrofia facetaría y osteofitos entre otros. Es de gran ayuda en estenorraquis asociada a escoliosis, especialmente por la posibilidad de hacer cortes en distintos planos y realizar reconstrucciones.

\section{Tratamiento}

Siempre el tratamiento inicial es conservador. Este debe ajustarse según el paciente, sus características demográficas, su cuadro clínico y las opciones de tratamiento disponibles localmente.

Este consiste en las siguientes medidas: Inicialmente se recomienda modificación en lo posible de actividades que desencadenan la sintomatología y un reposo relativo. Se debe indicar medicamentos analgésicos como paracetamol u opiáceos o bien antiinflamatorios como no esteroidales o corticoides en agudo por corto tiempo.

Se recomienda asociar un tratamiento de fisioterapia, aunque evidencia sólida que sustente este punto es escasa. Incluye compresas húmedas calientes, masoterapia, acupuntura, ultrasonido, TENS, tracción, ejercicios quiroprácticos entre otros.

Respecto a la actividad física se recomienda mantener un cierto nivel de actividad con ejerci- cios aeróbicos o deportes sin impacto, idealmente en flexión (bicicleta por ejemplo).

Otra alternativa recomendada, con bastante literatura al respecto, es la inyección epidural de esteroides. La infiltración foraminal selectiva de la o las raíces comprometidas se ha demostrado que tiene mejores resultados que la epidural no selectiva. Estas inyecciones presentan un efecto analgésico retrasando y en ocasiones evitando una eventual cirugía. En cuanto al efecto analgésico, se define como un alivio significativo del dolor cuando disminuye a menos de $50 \%$ del dolor inicial evaluado con EVA (escala visual análoga). Esto ocurre en 60 a 70\% de los pacientes por un período de 4 a 6 semanas en promedio. No se ha encontrado diferencia entre la infiltración con corticoides versus solución salina a los 3 meses $y$ en adelante en los estudios hechos al respecto. Según algunos estudios se evitaría la cirugía hasta en $50 \%$ de los casos y en los casos en los que no se evita se observa un retraso de la misma de entre 13 y 28 meses. Ahora, en caso que exista una buena respuesta a la inyección epidural del punto de vista del dolor (> 50\% alivio), existe $>90 \%$ de probabilidades que el dolor se alivie en forma significativa posterior a cirugía y que este alivio se mantendrá por más de 6 meses $^{11-16}$.

Se cree que la compresión crónica de las raíces puede producir un daño estructural en los nervios que puede ser menos reversible mientras más tiempo de evolución presente. Basado en lo anterior, Derby et a ${ }^{17}$ plantean que los resultados de la descompresión quirúrgica son mejores si el procedimiento es realizado antes de 1 año de iniciado el cuadro.

Respecto al tratamiento quirúrgico ${ }^{18-21}$, este tiene los objetivos de mejorar la calidad de vida del paciente, disminuir tanto el dolor lumbar como radicular y mejorar el déficit neurológico. Se plantea la cirugía cuando existe una falta de respuesta a tratamiento conservador por al menos 12 semanas asociado a una alteración significativa en la realización de actividades de la vida diaria objetivables con escalas de Oswestry Disability Index (ODI) y 36 Item Short Form General Health Survey (SF-36) $)^{22-23}$ y dolor radicular moderado o severo basado en el EVA.

Respecto al tipo de tratamiento quirúrgico, la descompresión pura ha sido el tratamiento de elección para la estenorraquis lumbar sintomática. El candidato ideal para una cirugía descompresiva 
pura es aquel con un dolor radicular predominante, con leve o sin dolor lumbar. Existen múltiples técnicas de descompresión, existiendo controversia respecto a cuál es la mejor técnica quirúrgica para cada caso: destaca la laminectomía clásica, laminotomía bilateral ("port hole tech"), laminoplastía, descompresión minimamente invasiva, técnica de Mc Culloch, entre otras. Independiente de cual sea la técnica quirúrgica el objetivo es disminuir la presión mecánica sobre el saco dural y las raíces, que están comprimidos principalmente por el engrosamiento de las partes blandas (ligamento amarillo y cápsula articular) y en menor medida por los osteofitos de las articulaciones facetarías artrósicas. La cirugía consiste en la resección de la lámina para que el canal raquídeo quede abierto hacia posterior, remover los osteofitos de las articulaciones facetaría como también la resección de las partes blandas como el ligamento amarillo y cápsula articular hipertrofiada (Figura 2). Con lo anterior se tiene que lograr ver el saco dural y las raíces libres en el receso lateral (zona más frecuentemente afectada) y se tiene que objetivar con instrumentos (palpadores) la salida sin resistencia de las raíces a través de la forámina. En una descompresión pura es fundamental no inestabilizar el segmento a operar para lo cual no se debe resecar más de $50 \%$ de la articulación facetaria ni la pars interarticularis como tampoco realizar una discectomía radical, ya que quedaría inestable y sería necesario realizar una artrodesis instrumentada. La resección de los ligamentos interespinosos no está demostrado que inestabilice el segmento, pero algunos autores recomiendan su conservación en la cirugía.

Respecto a cuándo realizar una artrodesis instrumentada hay numerosa evidencia en la literatura sobre sus indicaciones ${ }^{27-33}$. Estas comprenden principalmente la espondilolistesis degenerativa, la escoliosis degenerativa, los pacientes con alteración del balance sagital y coronal de la columna lumbar, recurrencia de la raquiestenosis en un paciente previamente descomprimido, estenosis adyacente a un segmento artrodesado y la consideración intraoperatoria del cirujano ${ }^{34-40}$.

Respecto a los resultados post-quirúrgicos, más de $80 \%$ de los pacientes refieren alivio sintomático a corto plazo (2 años). En pacientes seleccionados, con estenorraquis lumbar sintomática de más de 3 meses de evolución se ha observado un mejor resultado del tratamiento quirúrgico respecto al manejo conservador evaluados con escalas funcionales como el SF 36 y ODI a 4 años de seguimiento $^{19,20}$. Sin embargo, 7-10 años posterior a la cirugía al menos $1 / 3$ de los pacientes refieren reinicio de dolor lumbar. Los índices de reoperación a 10 años plazo van de los $10-23 \%{ }^{21,41}$.

Nuestra conducta en estenorraquis lumbar sintomática es siempre el manejo conservador en primera instancia ya que se sabe que un porcentaje superior al $50 \%$ de los pacientes responden satisfactoriamente al tratamiento no quirúrgico incluyendo analgésicos, kinesioterapia y/o infiltración con corticoides (epidurales o foraminales). El período de evaluación de la respuesta a un tratamiento conservador es de al menos 3 meses. Para objetivar la intensidad del dolor lumbar-radicular usamos el EVA y para el grado de limitación funcional ocupamos el ODI y el SF 36 . En caso de mala respuesta a lo anterior se plantea tratamiento quirúrgico. La técnica a utilizar dependerá de factores clínicos e imagenológicos asociados comentados previamente.

\section{Conclusiones}

La raquiestenosis lumbar es un cuadro clínico que debe tenerse en cuenta en todo paciente sobre la $5^{\circ}$ década de la vida, con dolor lumbociático uni o bi lateral. Debe ser estudiado con radiografías y resonancia magnética de columna lumbar. El manejo inicial es siempre conservador con analgésicos, kinesioterapia y eventualmente infiltración con corticoides epidurales o foraminales. Cuando el manejo médico no da buen resultado, persistiendo el paciente con un dolor radicular severo asociado a una limitación significativa en sus actividades de la vida diaria, es planteable la cirugía.

\section{Referencias}

1. Kalichman L, Cole R, Kim DH, Li L, Suri P, Guermazi A, et al. Spinal Stenosis Prevalence and association with symptoms: The Framingham Study. Spine J 2009; 9 (7): 545-50.

2. Dommisse GF. Morphological aspects of the lumbar spine and lumbosacral region. Orthop Clin North America 1975; 6: 163-75.

3. Dupuis PR, Yong-Hing K, Cassidy JD, Kirkaldy-Willis W. H. Radiologic diagnosis of degenerative lumbar spinal instability. Spine 1985; 10: 262-76. 
4. Garfin S, Herkowitz H, Mirkovic S. Spinal Stenosis, An Instructional Course Lecture, American Academy of Orthopaedic Surgeons. J Bone Joint Surg 1999; 81-A: 4.

5. Boden S, Wiesel S. Lumbar Spine Imaging: Role in Clinical Decision Making. J Am Acad Orthop Surg 1996; 4: 238-48.

6. Boden S. Current Concepts Review - The Use of Radiographic Imaging Studies in the Evaluation of Patients Who Have Degenerative Disorders of the Lumbar Spine. J Bone Joint Surg [Am] 1996; 78-A; 114-24.

7. Jensen MC, Brant-Zawadzki MN, Obuchowski N, Modic MT, Malkasian D, Ross JS. "Magnetic Resonance Imaging of the Lumbar Spine in People without Back Pain." N Engl J Med 1994; 331 (2): 69-73.

8. Boden SD, Davis DO, Dina TS, Patronas NJ, Wiesel SW. Abnormal magnetic-resonance scans of the lumbar spine in asymptomatic subjects. A prospective investigation. J Bone Joint Surg Am 1990; 72 (3): 403-8.

9. Amundsen, T, Weber H, Lileas F, Nordai HJ, Abdelnoor M, Magnaes B. "Lumbar Spinal Stenosis: Clinical and Radiologic Features." Spine 1995 20; 1178-86.

10. Coxon A, Shipley R, Murray M, Roper H, White S, Nagendar K, Greenough CG. Referred pain can radiate below the knee. European Spine Journal 2009; 18: 3.

11. DePalma M, Slipman C. Evidence-informed management of chronic low back pain with epidural steroid injections. The Spine Journal 2008; 8: 45-55.

12. Young IA, Hyman GS, Packia-Raj LN, Cole AJ. The Use of Lumbar Epidural/Transforaminal Steroids for Managing Spinal Disease. J Am Acad Orthop Surg 2007; 15 (4): 228-38.

13. Biyani A, Andersson GB. Low Back Pain: Pathophysiology and Management. J Am Acad Orthop Surg 2004; 12: 106-15.

14. Thomas E, Cyteval C, Abiad L, Picot MC, Taourel P, Blotman F. Efficacy of transforaminal versus interspinous corticosteroid injection in discal radiculalgia-a prospective, randomised, double-blind study. Clin Rheumatol 2003; 22: 299-304.

15. Riew KD, Yin Y, Gilula L, Bridwell KH, Lenke LG, Lauryssen C, et al. The effect of nerve-root injections on the need for operative treatment of lumbar radicular pain. A prospective, randomized, controlled, doubleblind study. J Bone Joint Surg Am 2000; 82-A (11): 1589-93.

16. Karppinen J, Malmivaara A, Kurunlahti M, Kyllönen E, Pienimäki T, Nieminen P, et al. Periradicular Infiltration for Sciatica, A Randomized Controlled Trial. Spine (Phila Pa 1976) 2001; 26 (9): 1059-67.

17. Derby R, Kine G, Saal JA, Reynolds J, Goldthwaite N, White $\mathrm{AH}$, et al. Response to steroid and duration of radicular pain as predictors of surgical outcome. Spine 1992; 17 (6 Suppl): S176-83.

18. Malmivaara A, Slätis P, Heliövaara M, Sainio P, Kinnunen H, Kankare J, et al. Finnish Lumbar Spinal Research Group. Surgical or nonoperative treatment for lumbar spinal stenosis? A randomized controlled trial. Spine (Phila Pa 1976) 2007; 32 (1): 1-8.

19. Weinstein JN, Tosteson TD, Lurie JD, Tosteson AN, Blood E, Hanscom B, et al. The SPORT Investigators. Surgical versus Nonsurgical Therapy for Lumbar Spinal Stenosis. N Engl J Med 2008; 358: 794-810.

20. Weinstein JN, Tosteson TD, Lurie JD, Tosteson A, Blood E, Herkowitz H, et al. The SPORT Investigators. Surgical versus Non operative treatment for lumbar spinal stenosis 4 years results of the Spine Patient Outcome Research Trial. Spine 2010; 35 (14): 1329-38.

21. Hansraj KK, Cammisa FP Jr, O'Leary PF, Crockett HC, Fras CI, Cohen MS. Decompression Surgery for Typical Lumbar Stenosis. Clin Orthop Relat Res 2001; 384: 10-7.

22. Fairbank JC, Couper J, Davies JB, O'Brien JP. The Oswestry low back pain disability questionnaire. Physiotherapy 1980; 66 (8): 271-3.

23. Ware JE Jr, Sherbourne CD. The MOS 36-item shortform health survey (SF-36). I. Conceptual framework and item selection. Med Care 1992; 30 (6): 473-83.

24. Atlas SJ, Keller RB, Wu YA, Deyo RA, Singer DE. Long-Term Outcomes of Surgical and Nonsurgical Management of Sciatica Secondary to a Lumbar Disc Herniation: 10 Year Results from the Maine Lumbar Spine Study. Spine 2005; 30 (8): 927-35.

25. Yuan PS, Booth RE Jr, Albert TJ. Nonsurgical and surgical management of lumbar spinal stenosis. J Bone Joint Surg (Am) 2004; 86: 2319-30.

26. Teo EC, Lee KK, Qiu TX, Ng HW, Yang K. The Biomechanics of Lumbar Graded Facetectomy under AnteriorShear Load. IEEE Trans Biomed Eng 2004; 51(3): 443-9.

27. Herkowitz HN, Kurz LT. Lumbar Spinal Stenosis: Indications for Arthrodesis and Spinal Instrumentation. AAOS Instr Course Lect 2005; 54: 313-9.

28. Mardjetko SM, Connolly PJ, Shott S. Degenerative Lumbar Spondylolisthesis: A Meta-Analysis of Literature 1970-1993. Spine 1994; 19 (20 Suppl): 2256S-65S.

29. Bridwell KH, Sedgewick TA, O'Brien MF. The role of fusion and instrumentation in the treatment of degenerative spondylolisthesis with spinal stenosis. J Spinal Disord 1993; 6: 461-72.

30. Zdeblick TA. A prospective randomized study of lumbar fusion. Spine 1993; 18: 983-91.

31. Herkowitz HN, Kurz LT. Degenerative lumbar spondylolisthesis with spinal stenosis: a prospective study comparing decompression with decompression and 
intertransverse process arthrodesis. J Bone Joint Surg Am 1991; 73: 802-8.

32. Fischgrund JS, Mackay M, Herkowitz HN, Brower R, Montgomery DM, Kurz LT. Volvo Award winner in clinical studies. Degenerative lumbar spondylolisthesis with spinal stenosis: a prospective, randomized study comparing decompressive laminectomy and arthrodesis with and without spinal instrumentation. Spine 1997; 22 (24): 2807-12.

33. Kornblum, MB, Fischgrund JS, Herkowitz HN, Abraham DA, Berkower DL, Ditkoff JS. Degenerative Lumbar Spondylolisthesis With Spinal Stenosis: A Prospective Long-Term Study Comparing Fusion and Pseudarthrosis. Spine 2004; 29 (7): 726-33.

34. Lenke LG, Edwards CC, Bridwell KH. The Lenke classification of adolescent idiopathic scoliosis: how it organizes curve patterns as a template to perform selective fusions of the spine. Spine 2003; 28: S199-S207.

35. Rinella A, Bridwell K, Kim Y, Rudzki J, Edwards C, Roh $\mathrm{M}$, et al. Late complications of adult idiopathic scoliosis primary fusions to L4 and above. The effect of age and distal fusion level. Spine 2004; 29 (3): 318-25.

36. Postacchini F, Cinotti G. Bone regrowth after surgical decompression for lumbar spinal stenosis. J Bone Joint Surg 1992; 74B: 862-9.

37. Lehmann TR, Spratt KF, Tozzi JE, Weinstein JN, Reinarz SJ, el Khoury GY, et al. Long-term follow up of lower lumbar fusion patients. Spine 1987; 12 (2): 97-104.

38. Dall BE, Rowe DE. Degenerative spondylolisthesis. Its surgical management. Spine 1985; 10: 668-72.

39. Abumi K, Panjabi MM, Kramer KM, Duranceau J, Oxland T, Crisco JJ. Biomechanical evaluation of lumbar spinal stability after graded facetectomies. Spine 1990; 15 (11): 1142-7.

40. Kleinstuck, FS, Grob D, Lattig F, Bartanusz V, Porchet F, Jeszenszky D, et al. The Influence of Preoperative Back Pain on the Outcome of Lumbar Decompression Surgery. Spine 2009; 34 (11): 1198-203.

41. Katz JN, Lipson SJ, Chang LC, Levine SA, Fossel AH, Liang MH. Seven-to 10-year Outcome of Decompressive Surgery for Degenerative Lumbar Spinal Stenosis. Spine 1996; 21 (1): 92-97. 